\title{
Gewisse Segalsche Algebren auf lokalkompakten Gruppen
}

\author{
Von \\ Dethev Poguntee
}

Bei den Untersuchungen über Symmetrie von Gruppenalgebren (insbesondere auflösbarer Liescher Gruppen) traten in natürlicher Weise gewisse Funktionenalgebren auf lokalkompakten (abelschen) Gruppen auf. Explizit wurden diese Algebren von Leptin in [3] studiert.

Im Rahmen der Winterschule 1979 über topologische Gruppen in Wien lernte ich dann die Konstruktion minimaler Segalscher Algebren auf abelschen Gruppen von Feichtinger kennen. Diese Konstruktion legte es nahe, auch die oben erwähnten Funktionenalgebren durch eine Minimalitätseigenschaft zu charakterisieren. Dieses soll nun in dem vorliegenden Aufsatz geschehen.

Zur Präzisierung zunächst die folgende

Definition 1. Sei $G$ eine lokalkompakte Gruppe. Eine selbstadjungierte Unteralgebra $U$ von $C_{\infty}(G)$, der Algebra aller komplexwertigen, im Unendlichen verschwindenden, stetigen Funktionen auf $G$, zusammen mit einer Norm ||$(\geqq|| \infty)$, heißt homogene Funktionen-Algebra auf $G$, wenn gilt:

(i) $(U, \mid)$ ist eine Banachsche Algebra.

(ii) $U$ ist invariant gegen Linkstranslationen, d.h. für jedes $x \in G$ liegt mit $\mathrm{u}$ auch $u^{x}$ in $U$, wobei $u^{x}$ durch $u^{x}(y)=u(x y)$ erklärt ist.

(iii) $|u|=\left|u^{*}\right|=\left|u^{x}\right|$ für alle $u \in U$ und alle $x \in G$.

(iv) $x \rightarrow u^{x}$ ist für alle $u \in U$ eine stetige Abbildung von $G$ in $U$.

(v) $U$ ist eine reguläre Funktionen-Algebra.

Beispiele solcher Algebren sind natürlich die Fourier-Eymardsche Algebra $A(G)$ für jede lokalkompakte Gruppe $G$, aber auch das folgende: Sei

$$
U=\{f \in A(\mathbb{R}) ; f(x)=0 \text { für } x \leqq 0\} .
$$

$U$ läßt sich als Unteralgebra von $C_{\infty}(\mathbb{R})$ auffassen vermöge der Einbettung $f \rightarrow \tilde{f}$, $\tilde{f}(x)=f\left(e^{x}\right)$. Diese Algebra $U$ tritt etwa beim Studium der $a x+b$-Gruppe auf, vgl. [2] oder auch [4] und [6].

Zu einer homogenen Funktionen-Algebra $U$ auf $G$ kann man die Leptinsche Algebra $L=L^{1}(G, U)$ bilden (bezüglich eines linken Haarschen Maßes auf $G$ ). Diese Algebra 
hat eine involutive Darstellung $\pi$ in $L^{2}(G)$, gegeben durch

$$
\{\pi(f) \xi\}(x)=\int_{G} f(y)\left(y^{-1} x\right) \xi\left(y^{-1} x\right) d y .
$$

Man kann nun die Frage stellen, wann $\pi(f)$ ein Operator vom Range 1 ist. In [3] wurde gezeigt: Definiert man für $a, b \in C_{\infty}(G)$ die Funktion $a \circ b: G \rightarrow C(G)$ durch

$$
(a \circ b)(x)(y)=\Delta(y) a(x y) \overline{b(y)} \text {, }
$$

wobei $\Delta$ die Modularfunktion von $G$ bezeichnet, und setzt man

$$
U_{1}=\{u \in U ; u \circ u \in L\},
$$

so gilt: Für ein $f \in L$ ist $\pi(f)$ dann und nur dann vom Range 1 , wenn es $u, v \in U_{1}$ mit $f=u \circ v$ gibt. $U_{1}$ ist von Null verschieden, genauer gilt:

Ist $u \in U_{0}$, d.h. $u \in U$ und der Träger von $u$, mit $\operatorname{Trg}(u)$ bezeichnet,

ist kompakt, so liegt

$$
u^{\prime}=\Delta^{-1} u \text { in } U_{1} \text {. }
$$

Beweis. Zunächst ist zu zeigen, daß $u^{\prime}$ in $U$ liegt. Dazu können wir natürlich $u \neq 0$ annehmen. Durch $s \rightarrow \bar{u}^{s} u^{s} u$ ist eine stetige Funktion von $G$ in $U$ mit kompaktem Träger definiert. Also existiert $v:=\int_{G} \bar{u}^{s} u^{s} u d s$ in $U$, und es gilt

$$
\begin{aligned}
v(x) & =\int_{\dot{G}} \bar{u}(s x) u(s x) u(x) d s=\int_{G} u(x) \Delta(x)^{-1} \bar{u}(t) u(t) d t \\
& =\Delta(x)^{-1} u(x)|u|_{2}^{2}=u^{\prime}(x)|u|_{2}^{2} .
\end{aligned}
$$

Mit $v$ liegt auch $u^{\prime}$ in $U$.

Weiter gilt $\left(u^{\prime} \circ u^{\prime}\right)(s)(y)=\Delta(y) u^{\prime}(s y) \overline{u^{\prime}(y)}=u^{\prime}(s y) \overline{u(y)}$ oder $\left(u^{\prime} \circ u^{\prime}\right)(s)=u^{\prime} s \tilde{u}$. Folglich liegen die Werte von $u^{\prime} \circ u^{\prime}$ in $U$, und da $u^{\prime} \circ u^{\prime}$ darüber hinaus eine stetige Funktion mit kompaktem Träger ist, liegt $u^{\prime} \circ u^{\prime}$ in $L=L^{1}(G, U)$.

Ferner wurde in [3] die folgende nützliche Beschreibung von $U_{1}$ gegeben.

Ist $w$ ein beliebiges, von Null verschiedenes Element in $U_{1}$, so gilt:

$$
U_{1}=\{a \in U ; w \circ a \in L\} \text {. }
$$

Wählt man speziell $w=u^{\prime}=A^{-1} u$ mit einem $u \in U_{0}, u \neq 0$, so ist

$$
(w \circ a)(s)=\Delta(s)^{-1} u^{s} \vec{a} \in U .
$$

Ein $a \in U$ liegt also dann und nur dann in $U_{1}$, wenn $w \circ a$ summierbar ist. Man erhält:

$$
\begin{aligned}
& \text { Ist } u \in U_{0}, u \neq 0, \text { so gilt } \\
& U_{1}=\left\{a \in U ; \int_{G} \Delta(s)^{-1}\left|u^{s} \bar{a}\right| d s<\infty\right\}
\end{aligned}
$$

oder

$$
U_{1}=\left\{a \in U ; \int_{G}\left|u \bar{a}^{s}\right| d s<\infty\right\}
$$


An dieser Beschreibung liest man auch $U_{0} \subset U_{1}$ ab. Die Gleichung (3) wurde in [3] zur Normierung von $U_{1}$ benützt, indem man $|a|_{1}=|w \circ a|_{L}\left(a \in U_{1}\right)$ setzt.Verschiedene $w$ 's führen zu äquivalenten Normen, und $U_{1}$ wird so zu einer Banachschen Algebra. Ist speziell $w=u^{\prime}\left(u \in U_{0}, u \neq 0\right)$, so setzen wir, um die Abhängigkeit von $u$ zum Ausdruck zu bringen:

$$
|a|_{u}:=\left|u^{\prime} \circ a\right|_{L}=\int_{G}\left|u \bar{a}^{s}\right| d s \quad\left(a \in U_{1}\right)
$$

||$_{u}$ ist also eine von den oben beschriebenen äquivalenten Normen auf $U_{1}$. Weiter gilt:

Satz 1. Sei $U$ eine homogene Funktionen-Algebra auf der lokalkompakten Gruppe $G$. Dann liegt $U_{0}$ dicht in $U_{1}$.

Beweis. Sei $u \in U_{0}, u \neq 0$, fest gewählt. O.B.d.A. können wir $u \geqq 0$ annehmen. Es ist zu zeigen, daß zu $\varphi \in U_{1}$ und $\varepsilon>0$ ein $\tilde{\varphi} \in U_{0}$ existiert mit

$$
|\varphi-\tilde{\varphi}|_{u} \leqq \varepsilon \text {. }
$$

Wir definieren $c>0$ durch

und seszen

$$
c^{-1}=\int_{G} u\left(t^{-1}\right) d t
$$

$$
\eta=\varepsilon\left\{1+c|u|\left|\operatorname{Trg}(u) \operatorname{Trg}(u)^{-1}\right|\right\}^{-1},
$$

wobei $|$.$| (auch) das linke Haarsche Maß bezeichnet. Laut Voraussetzung ist$

$$
\int_{G}\left|\varphi^{t} u\right| d t=\int_{G}\left|\varphi u^{t-1}\right| d t<\infty .
$$

Es gibt folglich eine kompakte Menge $L \subseteq G$ mit $L=L^{-1}$ und $\int_{G \backslash L}\left|\varphi u^{t^{-1}}\right| d t<\eta$. Sei nun $L^{\prime}:=L \operatorname{Trg}(u) \operatorname{Trg}(u)^{-1}$ und $\tilde{\varphi}:=c \int_{L^{\prime}} u^{s^{-1}} d s$. Offensichtlich ist $\tilde{\varphi} \in U_{0}$.

Weiter gilt

$$
\varphi u^{t}=\tilde{\varphi} u^{t} \text { für alle } t \in L .
$$

$\mathrm{Zu}$ zeigen ist also

$$
\varphi(x) u(t x)=\tilde{\varphi}(x) u(t x) \quad \text { für } \quad t \in L, \quad x \in G .
$$

Ist $t x \notin \operatorname{Trg}(u)$, so ist diese Gleichung trivial. Nehmen wir also $t x \in \operatorname{Trg}(u)$ an. Dann ist $x \in L \operatorname{Trg}(u)$.

$\mathrm{Zu}$ zeigen bleibt

$$
x \in L \cdot \operatorname{Trg}(u) \Rightarrow \varphi(x)=\tilde{\varphi}(x) .
$$

Nun ist

$$
\tilde{\varphi}(x)=c \int_{I^{\prime}} \varphi(x) u\left(s^{-1} x\right) d s=c \varphi(x) \int_{L^{\prime}} u\left(s^{-1} x\right) d s .
$$

Nach Konstruktion gilt nun: $s \notin L^{\prime} \Rightarrow s^{-1} x \notin \operatorname{Trg}(u)$ und folglich

also $\tilde{\varphi}(x)=\varphi(x)$.

$$
\int_{L^{\prime}} u\left(s^{-1} x\right) d s=\int_{G} u\left(s^{-1} x\right) d s=\int_{G} u\left(\left(x^{-1} s\right)^{-1}\right) d s=\int_{G} u\left(s^{-1}\right) d s=c^{-1},
$$


Schätzen wir nun $|\varphi-\tilde{\varphi}|_{u}$ ab: Nach Definition und wegen $\left(^{*}\right)$ gilt

$$
\begin{aligned}
|\varphi-\tilde{\varphi}|_{u} & =\int_{G}\left|(\varphi-\varphi) u^{t^{-1}}\right| d t=\int_{G \backslash L}\left|(\varphi-\tilde{\varphi}) u^{t^{-1}}\right| d t \\
& \leqq \int_{G \backslash L}\left|\varphi u^{t^{-1}}\right| d t+\int_{G \backslash L}\left|\tilde{\varphi} u^{t^{-1}}\right| d t .
\end{aligned}
$$

Der erste Summand ist $<\eta$ nach Wahl von $L$. Nach Definition von $\tilde{\varphi}$ gilt:

$$
\int_{G \backslash L}\left|\tilde{\varphi} u^{t^{-1}}\right| d t=c \int_{G \backslash L}\left|\int_{L^{\prime}} u^{t^{-1}} u^{s^{-1}} \varphi d s\right| d t
$$

Für festes $t$ ist

$$
\left|\int_{L^{\prime}} u^{t^{-1}} u^{s^{-1}} \varphi d s\right| \leqq \int_{G}\left|u^{t^{-1}} u^{s^{-1}} \varphi\right| d s \leqq \int_{t \operatorname{Trg}(u) \operatorname{Trg}(u)^{-1}}\left|u^{t^{-1}} u^{s^{-1}} \varphi\right| d s,
$$

da der Integrand außerhalb dieser Menge verschwindet.

Folglich gilt

$$
\left|\int_{L^{\prime}} u^{t^{-1}} u^{s^{-1}} \varphi d s\right| \leqq\left|u^{t^{-1}} \varphi\right||u|\left|\operatorname{Trg}(u) \operatorname{Trg}(u)^{-1}\right| .
$$

Damit ergibt sich

$$
\begin{aligned}
\int_{G \backslash L}\left|\tilde{\varphi} u^{t^{-1}}\right| d t & \leqq c|u|\left|\operatorname{Trg}(u) \operatorname{Trg}(u)^{-1}\right| \int_{G \backslash L}\left|u^{t-1} \varphi\right| d t \\
& \leqq c|u|\left|\operatorname{Trg}(u) \operatorname{Trg}(u)^{-1}\right| \eta
\end{aligned}
$$

und folglich

$$
|\varphi-\tilde{\varphi}| u \leqq \eta\left\{1+c|u|\left|\operatorname{Trg}(u) \operatorname{Trg}(u)^{-1}\right|\right\}=\varepsilon, \quad \text { q.e.d. }
$$

Im folgenden wollen wir für abelsche Gruppen $G$ noch eine andere Charakterisierung von $U_{1}$ geben. Dazu führen wir die folgende Definition ein:

Definition 2. Sei $G$ eine lokalkompakte Gruppe, $U$ eine homogene FunktionenAlgebra auf $G$ mit Norm | |. Ein Ideal $V$ in $U$ zusammen mit einer Norm | $v$ auf $V$ heißt Segalsche Algebra in $U$, wenn gilt:

$10 V$ ist abgeschlossen gegen Linkstranslationen, $\mid v$ ist translationsinvariant, d.h. $\left|f^{x}\right|_{V}=|f|_{V}$ für alle $x \in G, f \in V$.

$2^{\circ}(V,|| v)$ ist ein Banachscher Raum.

$3^{0}$ Es gibt positive Konstante $C, D$ mit

$$
|f| \leqq C|f|_{\nu} \text { und }|f g|_{\nu} \leqq D|f|_{V}|g| \text { für alle } f \in V, \quad g \in U
$$

$4^{0} \quad U_{0} \subset V$.

Bemerkung 1. Da $U$ eine reguläre Funktionen-Algebra ist und es folglich zu jedem Kompaktum $K$ in $G$ ein $u \in U_{0}$ mit $u \equiv 1$ auf $K$ gibt (vgl. [7], p. 19), sind die $U$ Norm und die $V$-Norm auf Funktionen, deren Träger in einem festen Kompaktum liegt, äquivalent. 
Bemerkung 2. Um den Zusammenhang zu ,klassischeren“" Definitionen von Segalschen Algebren herzustellen, stellen wir fest: Ist $U$ zusätzlich eine Wienersche Algebra im Sinne von [7], p. 22, und $V$ ein Ideal mit $1^{0}-3^{0}$, so ist $4^{0}$ äquivalent zur Tatsache, daß $V$ dicht in $U$ liegt (vgl. [7], p. 20).

Bemerkung 3. Ist $U$ eine homogene Funktionen-Algebra auf der lokalkompakten Gruppe $G$, so ist $U_{1}$ eine Segalsche Algebra in $U$. Dies wurde bereits in [3] festgestellt und folgt im übrigen unmittelbar aus (4).

Bemerkung 4. In [1] hat Feichtinger die minimale Segalsche Algebra in der FourierEymardschen Algebra beschrieben. Diese Konstruktion läßt sich auch für eine beliebige homogene Funktionen-Algebra $U$ durchführen. Für eine kompakte Menge $K$ in $G$ mit nicht-leerem Inneren setze man $U_{\min }:=\left\{f \in U\right.$; es gibt Folgen $a_{n}$ in $U$ und $t_{n}$ in $G$ mit

$$
\left.\operatorname{Trg}\left(a_{n}\right) \subset K, \sum_{n=1}^{\infty}\left|a_{n}\right|<\infty \text { und } f=\sum_{n=1}^{\infty} a_{n}^{t_{n}}\right\}
$$

$U_{\min }$ wird normiert, indem man $|f|_{\text {min }}$ gleich dem Infimum der $\sum_{n=1}^{\infty}\left|a_{n}\right|$ setzt, $a_{n}$ wie oben. $U_{\min }$ ist unabhängig von dem gewählten $K, \mid$ min hängt nur bis auf Äquivalenz von $K$ ab. Weiter ist $U_{\min }$ eine Segalsche Algebra in $U$ und offenbar die kleinste.

Der folgende Satz zeigt nun, daß für homogene Funktionen-Algebren $U$ auf abelschen Gruppen $G$ die Gleichung $U_{\min }=U_{1}$ gilt. $U_{1}$ ist damit charakterisiert (und das ist das Hauptziel dieses Artikels) als die kleinste Segalsche Algebra in $U$.

Satz 2. Sei $U$ eine homogene Funktionen-Algebra auf der lokalkompakten abelschen Gruppe G. Dann gilt $U_{\min }=U_{1}$.

Beweis. Man braucht nur $U_{1} \subset U_{\min }$ zu beweisen. Da $U_{0}$ in $U_{1}$ dicht liegt (Satz 1) genügt es, die Norm von $U_{\min }$ auf $U_{0}$ gegen die Norm von $U_{1}$ abzuschätzen. Auf Grund der bekannten Struktur kompakt-erzeugter abelscher Gruppen besitzt $G$ eine zu $\mathbb{R}^{n} \times K$ ( $K=$ kompakte Gruppe) isomorphe offene Untergruppe $H$. Wenn man die Norm | min gegen ||$u$ für Funktionen in $U_{0}$ mit Träger in $H$ abschätzen kann, so kann man dies auch für beliebige Funktionen in $U_{0}$ tun. Wir können also im folgenden $G=\mathbb{R}^{n} \times K$ annehmen.

Zunächst konstruieren wir eine passende Funktion $u \in U_{0}$, welche wir zur Beschreibung der Norm ||$u$ auf $U_{1}$ verwenden werden. Sei $v \in U_{0}, v \geqq 0$ und $v \neq 0$. Durch Mittelung über $K$ kann man annehmen, daß $v$ konstant auf Nebenklassen modulo $K$ ist. Weiter sei

$$
Q=\left\{\left(x_{1}, \ldots, x_{n}, 0\right) \in G \mid 0 \leqq x_{i} \leqq 1\right\} \text { und } u:=\int_{Q} v^{t} d t
$$

wobei $d t$ das Lebesguesche Maß auf $\mathbb{R}^{n}$ bezeichnet. Mit dieser Setzung gilt dann $\sum_{\alpha \in \mathbb{Z}^{n}} u^{\alpha}=$ const (punktweise, nur endlich viele Summanden sind jeweils von Null 
verschieden). Durch geeignete Abänderung von $v$ kann man diese (positive) Konstante zu 1 normieren.

Sei nun $f \in U_{0}$ und $N \in \mathbb{N}$ dazu so gewählt, daß mit

$$
D:=\left\{\left(t_{1}, \ldots, t_{n}\right) \in \mathbb{R}^{n} ;\left|t_{i}\right| \leqq N\right\}
$$

die Inklusion $D \times K \subset-\operatorname{Trg}(f)+\operatorname{Trg}(u)$ gilt. Dann ist

$$
|f|_{u}=\int_{G}\left|f u^{x}\right| d x=\int_{-N}^{N} \cdots \int_{-N}^{N}\left|f u^{\left(t_{1}, \ldots, t_{n}\right)}\right| d t_{1} \ldots d t_{n} .
$$

Dieses Integral schätzen wir nach unten durch Riemannsche Summen ab. Sei $\varepsilon>0$ gegeben. Dann gibt es $k \in \mathbb{N}$ mit

$$
|f| u+\varepsilon \geqq \sum_{\alpha \in(\mathbb{1} / k) \mathbb{Z}^{n}} \frac{1}{k^{n}}\left|f u^{\alpha}\right|
$$

(in der Tat ist die Summe natürlich endlich). Setzen wir

$$
f_{\alpha}:=f \frac{u^{\alpha}}{k^{n}} \quad \text { für } \quad \alpha \in \frac{1}{k} \mathbb{Z}^{n} \text {, so gilt } \sum_{\alpha \in(1 / k) \mathbb{Z}^{n}} f_{\alpha}=f \text {. }
$$

Verwendet man also in der Definition von $U_{\min }$ und $/ /$ min die kompakte Menge $\operatorname{Trg}(u)$, so gilt

$$
|f|_{\min } \leqq \sum_{\alpha \in(1 / k) \mathbb{Z}^{n}}\left|f_{\alpha}\right| \leqq|f| u+\varepsilon, \text { also }|f|_{\min } \leqq|f| u
$$

Im Zusammenhang mit idealtheoretischen Untersuchungen in der Gruppenalgebra der Heisenberg-Gruppe wurde von Leptin die Frage gestellt, ob für $U=A(\mathbb{R})$ der Raum $S(\mathbb{R})$ der Schwartzschen Funktionen in $U_{1}$ enthalten ist. Die entsprechende Frage für $A(\mathbb{R})_{\min }$ wurde später von Feichtinger aufgeworfen. Der folgende Satz gibt darauf eine positive Antwort.

Satz 3. Sei $U=A\left(\mathbb{R}^{n}\right)$. Dann ist der Raum $S\left(\mathbb{R}^{n}\right)$ der Schwartzschen Funktionen auf $\mathbb{R}^{n}$ enthalten in $U_{1}=U_{\text {min }}$.

Beweis. Mit $F: S\left(\mathbb{R}^{n}\right) \rightarrow S\left(\mathbb{R}^{n}\right)$ sei die Fouriertransformation bezeichnet, weiter sei $\Delta: S\left(\mathbb{R}^{n}\right) \rightarrow S\left(\mathbb{R}^{n}\right)$ der Laplacesche Operator, und $p: \mathbb{R}^{n} \rightarrow \mathbb{R}$ sei definiert durch

$$
p(x)=1+|x|^{2 n}=1+\left\{x_{1}^{2}+\cdots+x_{n}^{2}\right\}^{n} .
$$

Wir setzen

$$
C=\int_{\mathbb{R}^{n}} p(x)^{-1} d x \quad(<\infty) .
$$

Es ist zu zeigen, daß $\int_{\mathbb{R}^{n}}\left|\varphi \varphi^{t}\right|_{A} d t$ für jedes $\varphi \in S\left(\mathbb{R}^{n}\right)$ endlich ist.

Nun gilt für jedes

$$
\begin{aligned}
t \in \mathbb{R}^{n}:\left|\varphi \varphi^{t}\right|_{A} & =\left|F^{-1}\left(\varphi \varphi^{t}\right)\right|_{L^{1}}=\left|p^{-1} p F^{-1}\left(\varphi \varphi^{t}\right)\right|_{L^{1}} \\
& \leqq C\left|p F^{-1}\left(\varphi \varphi^{t}\right)\right|_{L^{\infty}} \leqq C\left|F\left(p F^{-1}\left(\varphi \varphi^{t}\right)\right)\right|_{L^{1}} \\
& =C\left|\left\{1+\left(-\Delta^{n}\right)\right\} \varphi \varphi^{t}\right|_{L^{1}} \leqq C\left[\left|\varphi \varphi^{t}\right|_{L^{1}}+\left|\Delta^{n} \varphi \varphi^{t}\right|_{L^{1}}\right] .
\end{aligned}
$$

Offenbar sind $\int_{\mathbb{R}^{n}}\left|\varphi \varphi^{t}\right|_{L^{2}} d t$ und $\int_{\mathbb{R}^{n}}\left|\Delta^{n} \varphi \varphi^{t}\right|_{L^{1}} d t$ endlich, womit der Satz bewiesen ist. 


\section{Literaturverzeichnis}

[1] H. Feichrivger, The minimal strongly character invariant Segal algebra. Preprint, Wien.

[2] H. LEPTIN, Lokalkompakte Gruppen mit symmetrischen Algebren. Symposia Math. 22, $267-281$ (1977).

[3] H. LEPTIN, On onesided harmonic analysis in non commutative locally compact groups. Preprint, Bielefeld 1978.

[4] H. Lefrtin and D. PoguntKe, Symmetry and nonsymmetry for locally compact groups. Erscheint im J. Funct. Anal.

[5] D. Pogdatke, Symmetry (or simple modules) of some Banach algebras. Preprint, Bielefeld 1979.

[6] D. Poguntke, Symmetry and nonsymmetry for a class of exponential Lie groups. Erscheint im J. Reine Angew. Math.

[7] H. Retxer, Classical Harmonic Analysis and Locally Compact Groups. Oxford 1968.

Eingegangen am 4. 5. 1979

Anschrift des Autors:

D. Poguntke

Fakultät für Mathematik der Universität

Postfach 8640

D-4800 Bielefeld 